\title{
The Application in Speed Sensor-less Based on BP Neural Network Optimized by Genetic Algorithm
}

\author{
Yi LIU ${ }^{\mathrm{a}, * 1}$, Guorong LIU ${ }^{\mathrm{b}, * 2}$ \\ ${ }^{a}$ Department of Information engineering institute, Xiangtan University, Xiangtan, Hunan, China \\ ${ }^{b}$ HuNan Institution of Engineering, HuNan Institution of Engineering, Xiangtan, Hunan, China
}

\begin{abstract}
This paper puts forward a strategy that the sensor-less being optimized by the intelligent algorithm applies in the system of direct torque control. Based on the BP neural network in the identification of DTC speed, it optimizes the BP neural network speed identifier by using generic algorithm. It is proved by the simulation results that the neural network has been greatly improved by the application of intelligent algorithm, and the speed sensor-less based on the intelligent algorithm has better stability properties.
\end{abstract}

Index Terms: Direct Torque Control; Speed Sensor-less; BP Neural-Network; Genetic Algorithm

(C) 2011 Published by MECS Publisher. Selection and/or peer review under responsibility of the Research Association of Modern Education and Computer Science.

\section{Introduction}

Direct Torque Control (DTC) technology since its inception, receives universal concern for its innovative thinking, excellent dynamic and static characteristics. The speed loop in the high-performance AC speed control system is essential[1]. In order to achieve the feedback control of the motor speed and position, the speed sensor need to be installed in the motor-side[2]. However, this method increases the cost to the system and the volume, while reducing the reliability. Due to its complexity, the speed of identification of the AC speed control system has been a thorny issue. With the rapid development of intelligent algorithm, sensorless technology has been greatly improved. Fuzzy logic control, artificial neural network and a series of intelligent control theory has been widely applied. As the most developed, the BP neural network itself, has a lot of defects[3]. Therefore, we use the genetic algorithms to optimize the structure and weights of the BP networks, which improves the prediction accuracy of the network, and the accelerated convergence rate, to overcome the shortcomings of traditional forecasting methods. Simulation results show that the speed identification is much better.

\footnotetext{
* Corresponding author:

| E-mail address: ${ }^{* 1}$ tyhly500@163.com; ${ }^{* 2}$ lgr@ hnie.edu.cn
} 


\section{The Basic Equation of Direct Torque Control}

The mathematical model of the AC asynchronous motor in stationary coordinate system as follow:

\section{Voltage equation:}

$$
\left[\begin{array}{c}
u_{s \alpha} \\
u_{s \beta} \\
0 \\
0
\end{array}\right]=\left[\begin{array}{cccc}
R_{s}+L_{s} p & 0 & L_{m} p & 0 \\
0 & R_{s}+L_{s} p & 0 & L_{m} p \\
L_{m} p & \omega_{r} L_{m} & R_{r}+L_{r} p & \omega_{r} L_{r} \\
-\omega_{r} L & L_{m} p & -\omega_{r} L & R_{r}+L_{r} p
\end{array}\right]\left[\begin{array}{c}
i_{s \alpha} \\
i_{s \beta} \\
i_{r \alpha} \\
i_{r \beta}
\end{array}\right]
$$

\section{Flux linkage equation:}

$$
\left[\begin{array}{l}
\Psi_{s \alpha} \\
\Psi_{s \beta} \\
\Psi_{r \alpha} \\
\Psi_{r \beta}
\end{array}\right]=\left[\begin{array}{cccc}
L_{s} & 0 & L_{m} & 0 \\
0 & L_{s} & 0 & L_{m} \\
L_{m} & & L_{r} & \\
& L_{m} & & L_{r}
\end{array}\right]\left[\begin{array}{l}
i_{s \alpha} \\
i_{s \beta} \\
i_{r \alpha} \\
i_{r \beta}
\end{array}\right]
$$

In the formula, various quantities meaning is as follows:

$u_{s} 、 i_{s}$ stand for the stator -axis voltage and current

$\mathrm{u}_{\mathrm{s}} 、 \mathrm{i}_{\mathrm{s}}$, stand for the stator -axis voltage and current

$i_{r} 、 i_{r}$. stand for the rotor -axis voltage and -axis current

$R_{s} 、 L_{s} \quad$ stand for the resistance and inductance of the stator windings

$R_{r} 、 L_{r}$ stand for the resistance and inductance of the rotor windings

$\mathrm{L}_{\mathrm{m}}$ stands for mutual inductance between stator and rotor windingsP For derivative operator

P stands for the Differential operator

r $\quad$ stands for the Rotor electrical angular velocity

$\Psi_{s \alpha} 、 \Psi_{s \beta}$ stand for the stator -axis flux and -axis flux

$\Psi_{r \alpha} 、 \Psi_{r \beta}$ stand for the rotor -axis flux and -axis flux

Based on the voltage equation!̣and Flux equation (2), we can get the speed expression:

$$
\omega_{r}=\frac{L_{r}\left(u_{s \beta}-R_{r} i_{s \beta}\right)-\left(L_{s} L_{r}-L_{m} L_{m}\right) p i_{s \beta}+R_{r} \int\left(u_{s \beta}-R_{s} i_{s \beta}\right) d t-R_{r} L_{s} i_{s \beta}}{\left(L_{m} L_{m}-L_{s} L_{r}\right)+L_{r} \int\left(u_{s \alpha}-R_{s} i_{s \alpha}\right) d t}
$$




$$
\omega_{r}=\frac{L_{r}\left(u_{s \alpha}-R_{r} i_{s \alpha}\right)-\left(L_{\delta} L_{r}-L_{m} L_{m}\right) p i_{s \alpha}+R_{r} \int\left(u_{s \alpha}-R_{s} i_{s \alpha}\right)-R_{r} L_{s} i_{s \alpha}}{\left(L_{m} L_{m}-L_{s} L_{r}\right)+L_{r} \int\left(u_{s \beta}-R_{s} i_{s \beta}\right) d t}
$$

From the formula we can clearly see that, among the Motor speed ${ }_{r}$ and Stator voltage、Stator current there is one kind of extremely complex misalignment mapping relationship[4].

\section{The Design of Genetic Algorithm Optimizes the BP Speed Identification}

As shown in Fig. 1, the genetic algorithm which optimizes the BP neural network including the BP neural network's determination, the genetic algorithm optimization and the BP neural network forecast three parts.

We can clear see, the determination of the BP neural network includes definition network topology and initializing network weight and threshold value[5]. By identifying the input, output, hidden layer nodes we can determine the network topology and randomly initialize the weights and threshold value for the following steps for genetic algorithm. By selection, crossover and mutation of a series of operations, genetic algorithm optimization obtains the optimal weights and thresholds. Finally after the most superior weight and the threshold value are substituted in the BP neural network structure, we can carry on the training with the collected data, then obtaining the optimized result.

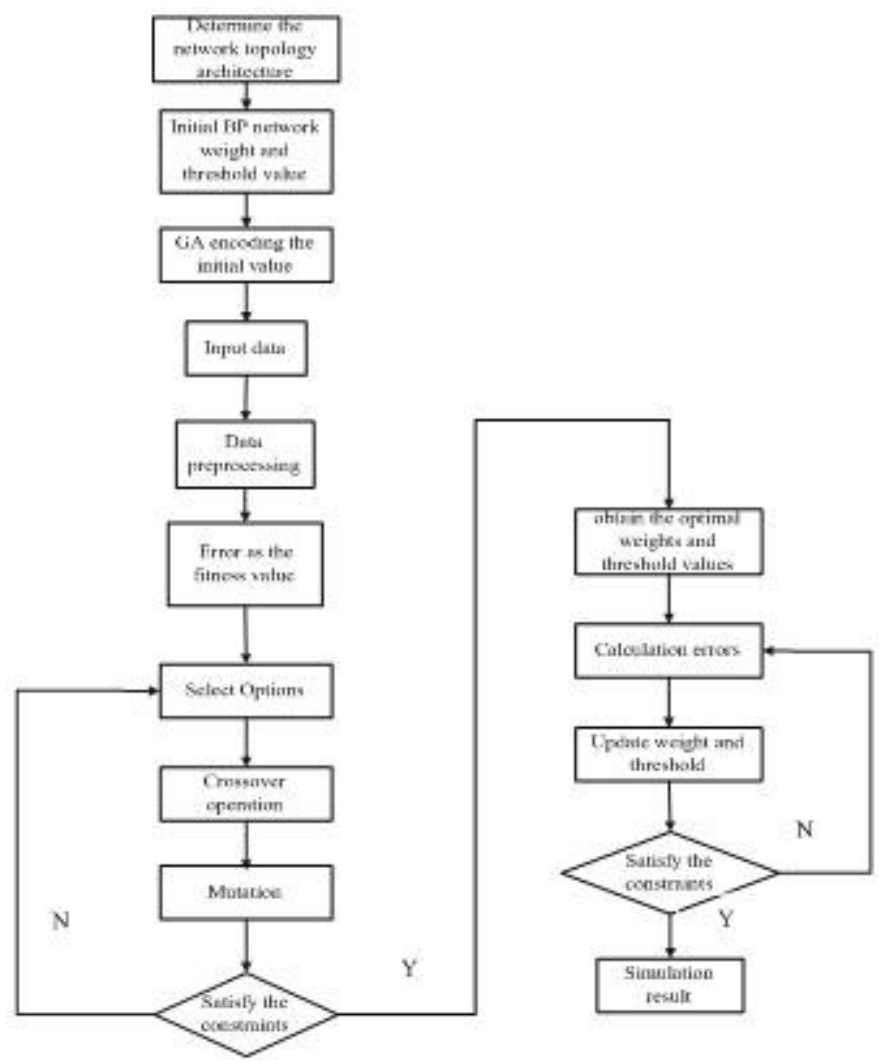

Fig. 1. GA optimize BP Neural Network Flow chart 


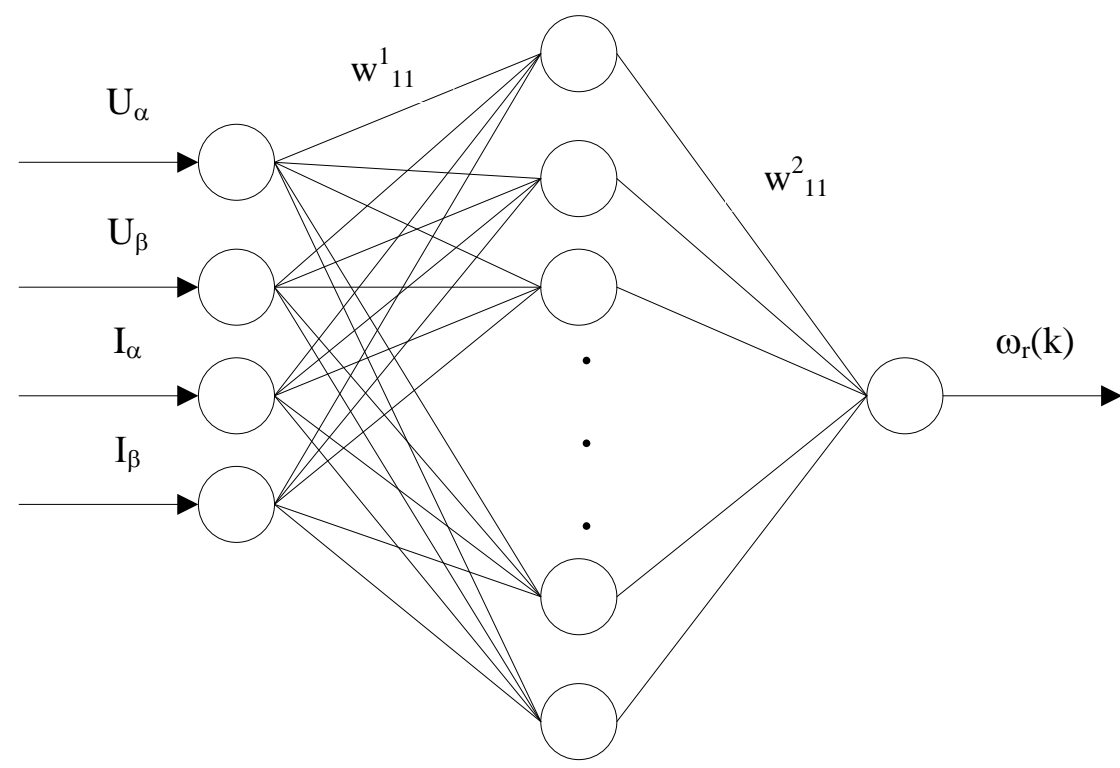

Fig. 2. Neural network speed identification network architecture

\subsection{BP Neural Network Design}

As the most developed BP neural network, S-function multi-layer BP neural network can realize any nonlinear mapping from input to output, as long as there are enough hidden layer units. This thesis selects three BP neural network constitution speed observation model, by the formula (3) and formula (4) determining the neural network structure for the four-input single-output.

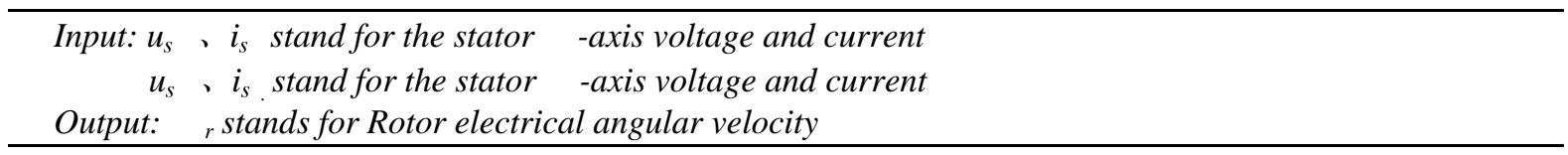

In BP neural network structure setup procedure, input layer nodes, hidden nodes and output layer nodes were set as follow:4,16,1.The evolution number of times is 100 times, and we select the learning rate as 0.1 , end of the target goal choose as 0.00001 . At this point in the BP neural network is used to build computing the cyclic fitness value.

BP neural network is not a perfect theory, and there are many defects in it: Multi-parameter optimization, which leads to slow convergence, it is difficult to achieve real time control; Objective function is a complex hypersurface and may contain multiple extreme points, which is easy to fall into local minimum. The initial value is set and has great impact on the training results, General rule of thumb and trial is difficult to obtain the optimal initial weights and thresholds. BP neural network initial weights and threshold values randomly, leading neural networks easy to fall into local optimum; the learning and the memory have the instability.

Construction of neural network use the function newff, because initialize the weights of BP, the threshold is random. Therefore, the genetic algorithm finds the optimal weights, the threshold. Then start the neural network training. 


\subsubsection{BP Neural Network Study Algorithm}

Neural networks can represent as any nonlinear function. The three-layer neural network output is calculated as follows:

\section{a) Calculation of the hidden layer output}

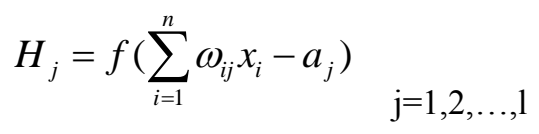

In the formula, ij is the weight of between the input the layer and hidden layer connection.a is the threshold hidden layer neuron, and 1 for the hidden nodes. $\mathrm{f}$ is hidden layer activation function. There are many expression forms of the function. Here we choose: $f(x)=\frac{1}{1+e^{-x}}$.

\section{b) Calculation of the output layer output}

According to the hidden layer output $\mathrm{H}$, Connection weights ${ }_{i k}$ and threshold $\mathrm{b}$, we predict the output of BP Neural network:

$$
O_{k}=\sum_{j=1}^{l} H_{j} \omega_{j k}-b_{k}{ }_{\mathrm{k}=1,2, \ldots, \mathrm{m}}
$$

\section{c) Error calculation}

According to neural network output $\mathrm{O}$ and desired output $\mathrm{Y}$, we can computing network prediction error e.

$$
e_{k}=Y_{k}-O_{k} \quad \mathrm{k}=1,2, \ldots, \mathrm{m}
$$

\section{2. $G A$}

As a fast, effective global optimization method, Genetic algorithm has obtained more and more widespread application. Establishment in natural selection and natural genetics foundation, the nature of the "survival of the fittest" theory of biological evolution introduced in the optimization of parameters of a code of series groups. In accordance with the fitness function through genetic selection, crossover and mutation screened individuals, so that the good fitness individual can be retained and individuals with poor fitness are eliminated. New groups not only inherited the previous generation of information, and better than the previous generation. In this paper, choosing the genetic algorithm to optimize the weights of neural networks, which is to optimize the neural network's weight and the threshold.

The genetic algorithm optimizes the BP neural network include population initialization, sufficiency function, selecting operation, crossover operation and Mutation. 


\subsubsection{Population Initialization}

Individual is encoded as real-coded and each individual string is a real number, which contain four parts :the input layer and hidden layer connection weights, hidden layer threshold, the hidden layer and output layer connection weights and the output layer threshold value. The individual contains all the neural network weights and thresholds. In the case of a network structure has been known, we can form a neural network structure which weight and the threshold are to be determined. Because the BP network structure is: input layer 4 nodes, hidden layer 16 nodes, output layer 1 nodes. A total of 80 weight, 17 threshold. Therefore, the individual coding length of the genetic algorithm is 97. The number of Evolution iterations chooses 100 and population size chooses 20. Crossover probability is 0.1 and mutation probability is 0.05 .

\subsubsection{Fitness function}

According to the initial value of the individual and the threshold based on the individual, with the training data after training BP neural network prediction system output. The predicted output and the absolute error between the desired output and $\mathrm{E}$ as the value of individual fitness $\mathrm{F}$ the absolute error $\mathrm{E}$ between the predicted output and the desired output as the individual fitness value F, The formula is:

$$
F=k\left(\sum_{i=1}^{n} a b s\left(y_{i}-o_{i}\right)\right)
$$

In the formula, $\mathrm{n}$ is the neural network output nodes; $\mathrm{y}_{\mathrm{i}}$ is the ith node expectation output; $\mathrm{o}_{\mathrm{i}}$ is the ith node forecast output; $\mathrm{k}$ is the coefficient parameter. The smaller the value of individual fitness, the more gifted individuals is.

\subsubsection{Select Options}

Select Options from the old population with a certain probability of selection to a new population of individual. The probability of an individual be chosen has a relevant to the fitness value. The smaller the value of individual fitness is, the greater the probability of being selected. Genetic algorithms select options has roulette method, tournament method and other methods. This topic chose roulette method, which is based on the proportion of fitness selection strategy. The probability $\mathrm{p}_{\mathrm{i}}$ of each individual $\mathrm{i}$ is:

$$
\begin{gathered}
f_{i}=k / F_{i} \\
p_{i}=\frac{f_{i}}{\sum_{j=1}^{N} f_{j}}
\end{gathered}
$$

In the formula, $\mathrm{F}_{\mathrm{i}}$ is the fitness of individual $\mathrm{i}$. Because the smaller fitness value the better the individual is. Therefore, individual selection before seeking reciprocal fitness value; $\mathrm{k}$ is the coefficient parameter; $\mathrm{N}$ is the number of individuals for the population. 


\subsubsection{Crossover Operation}

Crossover operation is by selecting two individuals from the individual, through the exchange of the two chromosomes to produce a new combination of excellent individuals. Because individuals with real number coding, so cross-operator method with real cross-Law,K-chromosome $a_{k}$ and L-chromosome $a_{1}$ in bit $j$ in the crossover operation as follows:

$$
\begin{aligned}
& a_{k j}=a_{k j}(1-b)+a_{l j} b \\
& a_{l j}=a_{l j}(1-b)+a_{k j} b
\end{aligned}
$$

In the formula, $\mathrm{b}$ is $[0,1]$ random number.

\subsubsection{Mutation}

Mutation choose an individual from the population and select the mutation point in the chromosome to produce better individuals. Selected i-individual's $\mathrm{j}$-genes $\mathrm{a}_{\mathrm{ij}}$ to mutate. Mutation operation is as follows:

$$
a_{i j}= \begin{cases}a_{i j}+\left(a_{i j}-a_{\max }\right) * f(g) & r \geq 0.5 \\ a_{i j}+\left(a_{\min }-a_{i j}\right) * f(g) & r<0.5\end{cases}
$$

In the formula, $a_{\max }$ is the upper bound for the gene $a_{i j} ; a_{\min }$ is the lower bound of the gene $a_{i j} ; f(g)=r_{2}(1-$ $\mathrm{g} / \mathrm{Gmax}) ; \mathrm{r}_{2}$ is a random number; $\mathrm{g}$ is the current number of iterations; $\mathrm{G}_{\max }$ is the maximum number of evolution; $\mathrm{r}$ is a random number between 0 and 1 .

\section{Simulations and Experimental Result}

\subsection{Data Acquisition}

We use the VB 6.0 to design a data acquisition run in the APC. Every time press the button "start sample" and the acquisition can get the 199 groups data through the RS232 interface.We can get the data when the

$=10 \mathrm{r} / \mathrm{s}, 20 \mathrm{r} / \mathrm{s}, 30 \mathrm{r} / \mathrm{s}, 40 \mathrm{r} / \mathrm{s}$ and $50 \mathrm{r} / \mathrm{s}$ and save the sampled data. 


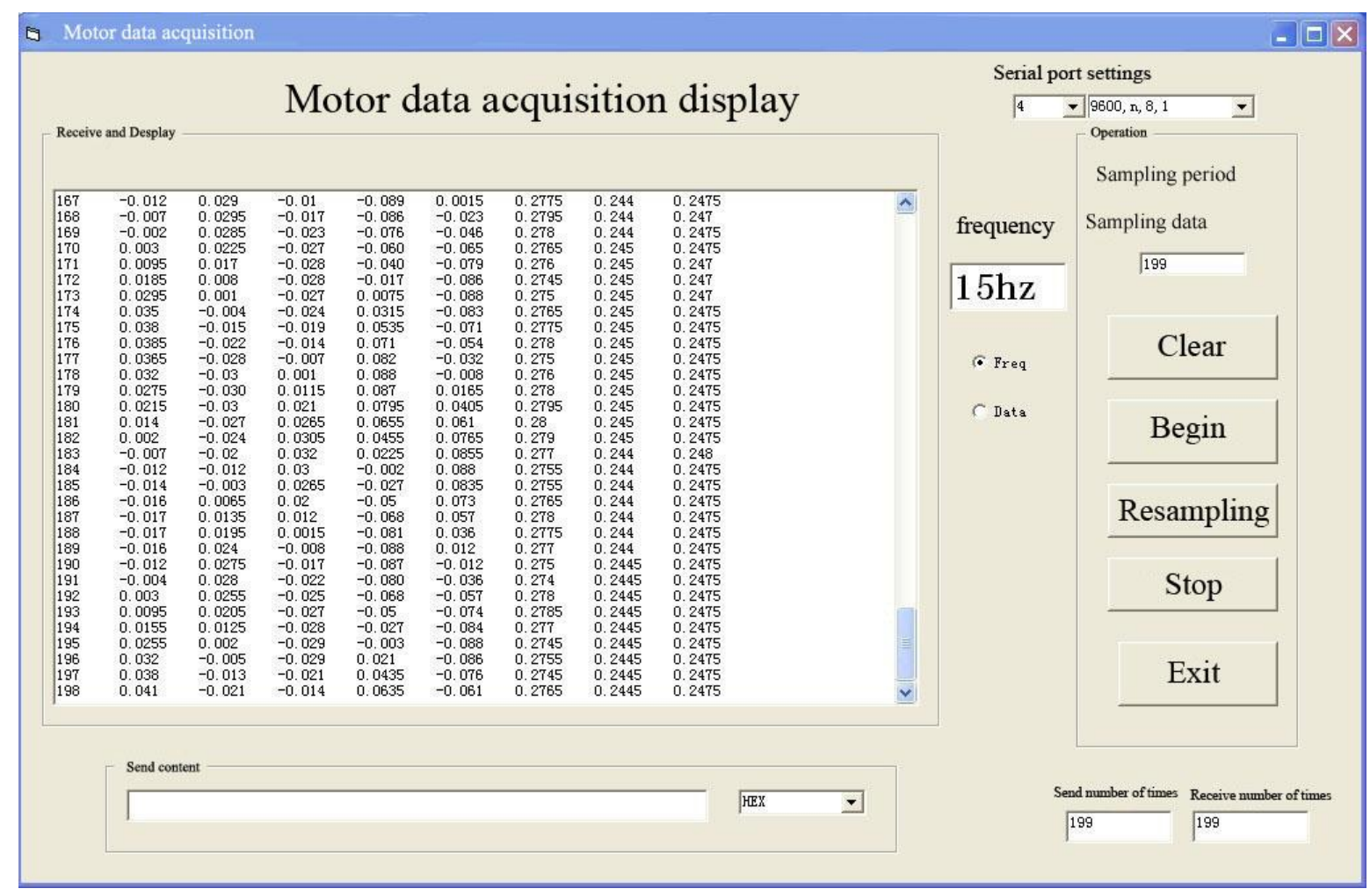

Fig. 3. Interface of on position machine data acquisition

\subsection{Simulation}

Carried out in the MATLAB simulation environment, we construct a four input one output of BP neural network. Data is used normalization and anti-normalization processing, transfer function of network nodes are used 'tansig' function. With a total of 199*5 which is 995 sets of data, we select 750 of them used to train the neural network. The remaining 245 sets of data is test data. Neural network by genetic algorithm optimization, obtaining the optimal weights and threshold values, with the optimized weights and thresholds to train BP neural network. Simulation results show that: Genetic algorithm optimized neural network has the ability to get more good speed identification. 


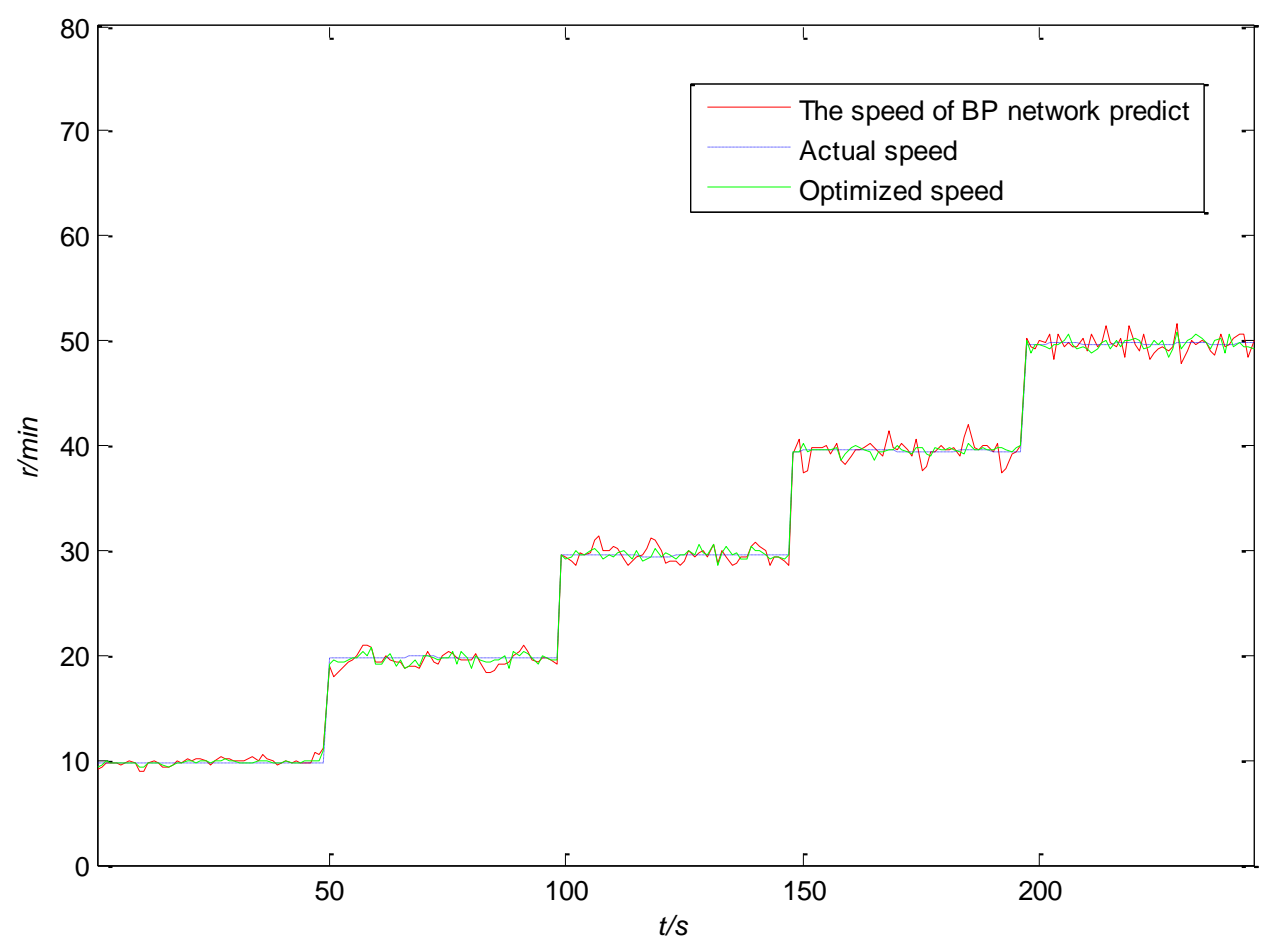

Fig. 3. BP neural network and genetic algorithm for the optimized rendering speed observer

Simulation results shown as above, in the motor speed under high speed and low speed, neural network has very good speed tracking performance; and the genetic algorithm optimized through the BP neural network has better tracking performance.

\section{Conclusion}

This article established a speed identifier based on the BP neural network. The application of genetic algorithm optimizes neural network design and the neural network structure. Speed up the convergence rate of neural network so to avoid the problem into minimum. Simulation results show that neural network algorithm to optimize the speed of identification has the ability of intuitive, also the high accuracy and provide a new method for the speed identify in sensor less direct torque control system. The experimental results show that the correctness of the theoretical analysis and the control strategy, and that using a intelligent algorithm in speed sensorless direct torque control system has better stability properties.

\section{Acknowledgements}

Supported by Hunan Natural Science Fund(09JJ8006). 


\section{References}

[1] Jun Liu, PuSheng Wu. "Application of fuzzy control in direct torque control of permanent magnet synchronous motor". [C] .IEEE intelligent control and Automation, 2004(5):4573-4576.

[2] Hisao Kubota, etc, "Speed Sensorless Field-Oriented Control of Induction Motor with Rotor Resistance Adaptation" [J] .IEEE trans. Industry. Application, September/October 1994, 3(5):1219-1224.

[3] Hecht-Nielson Robert.Theory of the Back Propagation Network[J]. IJCHNN(S01CH37222), 1989, 2(1): 583-604

[4] Geng Yang and Tung-hai Chin, "Adaptive Speed Identification Scheme for a Inverter-Induction Motor Drive",IEEE Transactio.Industry Applied.1993. 29(4):820-825

[5] K.S.Narendra and S.mukhopadhyay, "Intelligent Control Using Neural Networks", IEEE, Control Systems Magazine,pp. 11-1 8,April, 1992 